J. Dairy Sci. 93:6055-6063

(c) American Dairy Science Association, 2010.

\title{
Journal of Dairy Science Editorial Board and Ad Hoc Reviewers, 2010
}

The American Dairy Science Association and the Journal of Dairy Science gratefully acknowledge the following individuals who have served as members of the editorial board and as ad hoc reviewers in 2010.

Zaphiris Abas

Ali Abbassy

Saber Abd-Allah

Gamal Abdel-Azim

Analia Abraham

Ibrahim Abubakar

Lucia Abuquerque

Paolo Accornero

Margherita Addis

Adesogan Adesogan

Koushik Adhikari

Shantanu Agarwal

Ignacio Aguilar

Amin Ahmadzadeh

Patricia Aikman

R. Michael Akers

Saeed Akhtar

Elena Albanell

Marzia Albenzio

Jim Aldrich

Miguel Alvarez

Sergio Alvarez

Ibrahim Aman

Divakar Ambrose

Peter Amer

Burim Ametaj

Lourdes Amigo

Jonathan Amory

Arturo Anadon

Jens Bech Andersen

Kevin Anderson

Geert André

Sheila Andrew

Skelte Anema

Aikaterini Angelopoulou

Takayoshi Aoki

Ylva Ardö

Carlos Arechiga

Gastón Ares

Anastasio Arguello

Amichai Arieli

Louis Armentano

Valérie Arnould

Juan Jose Arranz
John Arthington

Kayanush Aryana

Kozo Asano

Melissa Ashwell

Joerg Aurich

Leonel Avendano-Reyes

Eman Ayad

Osama Azawi

Alex Bach

Lokenga Badinga

Christine Baes

Alessandro Bagnato

Lorna Baird

Ransom Baldwin

Vasileios Bampidis

T. C. Bamunuarachchige

Douglas Bannerman

Nidhi Bansal

David Barbano

Daniela Barile

Herman Barkema

Zoe Barker

John Barlow

Michael Barnes

Phil Barnes

Ciro Barros

Eric Bastian

Catherine Bastin

Howard Bateman

Lance Baumgard

Craig Baumrucker

Roswitha Baumung

David Beede

Donald Beitz

Nora Bello

Ronald Belyea

Christian Bendixen

Daniel Ben-Ghedalia

Torben Bennedsgaard

Gary Bennett

Jörn Bennewitz

Brian Bequette

Anna Berge

P. Berger
Roy Berghaus

Christer Bergsten

Bajram Berisha

Amiel Berman

Umberto Bernabucci

John Bernard

Donagh Berry

Sarah Berry

Steven Berry

Giuseppe Bertoni

Wagner Beskow

Thomas Besser

Greg Bethard

Petrus Beukes

Jeffrey Bewley

Rodrigo Bicalho

Christopher Bidwell

Stefano Biffani

Piter Bijma

Mario Binelli

Ana Binetti

Massimo Bionaz

Stephen Bishop

Dominique Blache

Dale Blasi

Donald Blayney

Kenneth Blemings

Elliot Block

Juerg H. Blum

Gerd Bobe

Jamie Boehmer

Charles Boeneke

Jarmila Bohmanova

Didier Boichard

Yves Boisclair

Mike Boland

Tommy Boland

Heinrich Bollwein

Adriana Bonanno

Daniela Borda

Stephanie Bordenave-Juchereau

Giorgio Borreani

Arjen Bot

Cynthia Bottema 
Kamal Bouarab

Sarah Boucher

H. Bovenhuis

Joan Bowen

Laura Boyle

Barry Bradford

Andrew Bradley

Ada Braghieri

Adriano Brandelli

Randall Brandsma

John Brantley

Martin Braunschweig

David Bravo

David Bray

James Breen

Gerhard Breves

Allen Bridges

J. Renaldi Brito

Jack Britt

Michel Britten

Glen Broderick

Sue Brotherstone

Keith Bryan

Solange Buchin

Frank Buckley

Ray A. Bucklin

Mateo Budinich

Albert (Bart) Buitenhuis

Marisa Bunning

Chris Burke

Edward Burnside

Betty Burri

D. Burrin

Christian Burvenich

Ron Butler

Stephen Butler

Andrea Cabiddu

Victor Cabrera

Margherita Caccamo

Keith Cadwallader

Roger Cady

Yimin Cai

Gerardo Caja

Luigi Calamari

Christina Caldari-Torres

Todd Callaway

Lien Callewaert

Sergio Calsamiglia

Mario Calus

Bruce Campbell

Taissa Canaes

Christopher Canale

Fabiola Canavesi

Antonello Cannas
Raul Cano

Rodolpho Canseco

John Cant

Rodolfo Cantet

Jude Capper

Anthony Capuco

Anna Caroli

Guillermo Carrillo-Castaneda

Maria Dolores Carro

Antonello Carta

Craig Carter

Serge Casaregola

David Casper

Martino Cassandro

Bennet Cassell

Lorena Castillejos

Alejandro Castillo

Manuel Castillo

Héctor Castillo-Juárez

Sara Casu

Alejandro Ceballos

Pietro Celi

Mario Ceron Munoz

Mireille Chahine

Claude Champagne

Marie-Christine Champomier-

Verges

Nuria Chapinal

Gemma Charlton

Larry Chase

Frederique Chaucheyras

Ricardo Chebel

Shuxing Chen

John Chenault

Debbie Cherney

Jacques Chesnais

Stefania Chessa

Hugh Chester-Jones

Mikhail Chickindas

Munashe Chigerwe

Yves Chilliard

Babu Chinnasamy

Jean-Marc Chobert

P. Yvan Chouinard

Giorgos Christodoulopoulos

Stephanie Clark

Wayne Coblentz

Michael Coffey

Tim M. Cogan

Patti Coggins

Wendie Cohick

John Cole

Robert Collier

Michael Collins
Dario Colombatto

David Combs

Giuseppe Comi

Jo Conington

Timothy Connelly

Erin Connor

Peter Constable

Nigel Cook

Wouter Coppieters

Benjamin Corl

Agnès Cornu

Milena Corredig

Georges Corrieu

Annamaria Costa

Elvira Costell

Josie Coverdale

A. Morrie Craig

Gerardus Cramer

Paola Cremonesi

Denny Crews

Brian Crooker

James Croom

Mark Crowe

Christian Cruywagen

Adriano Cruz

Kevin Cummings

John Currin

Monica Cutrignelli

Hans Daetwyler

Birol Dag

Robert Dailey

Joseph Dalton

Daalkhaijav Damiran

Kristy Daniels

Heather Dann

Ross Davidson

Stephen Davis

Michael Day

Maria De Angelis

Yvette De Haas

Peter De Kok

Dirk-Jan De Koning

Massimo De Marchi

Pasquale De Palo

Anne Marie de Passillé

Sander De Roos

Alexandre De Souza

Albert DeVries

Nicola Decaro

Chad Dechow

Hilton Deeth

Jeffrey Defrain

Fred Degraves

Laure Deguillaume 
Margaret Deigton

Mel Dejarnette

Jack Dekkers

Rosa Del Campo

Jeannine Delwiche

D. Mike Denbow

Carol Denysschen

Fabio Derensis

Marcela Derezende-Costa

Sarne Devliegher

Trevor Devries

Richard Dewhurst

Qiyu Diao

Alfredo Dicostanzo

Ellen Dierenfeld

Francisco Diez-Gonzalez

Jan Dijkstra

Nicolas Dilorenzo

Page Dinsmore

Michael Diskin

Ottmar Distl

Hilary Dobson

Joerg Dodenhoff

Lorraine Doepel

Ian Dohoo

Rutz Donald

Shawn Donkin

Catherine Donnelly

Larry Douglass

Mark Douglass

Chrysostomos Dovas

Rebecca Doyle

Maurice Doyon

James Drackley

Catalin Dragomir

MaryAnne Drake

Marc Drillich

Tom Druet

Jocelyn Dubuc

Todd Duffield

Susan Duncan

Tri Duong

Emilia Duranti

Lisa Durso

John Dyer

Robert Dyer

Alan Ealy

Maurice Eastridge

Misty Abrams Edmondson

Tom Edrington

J. Lannett Edwards

Monika Ehling-Schulz

James Ehrlich

Susan Eicher
Fatma Ekinci

Osman El Owni

Ibtisam E. M. El Zubeir

Gaber El-Baradei

Kathryn Ellis

Ahmed Elmoslemany

Theodore Elsasser

M. M. Eltholth

Francois Elvinger

Mauricio Elzo

Ulf Emanuelson

Rodrigo Emediato

Marcia Endres

Ola Engelsen

Terry Engle

Francis Enjalbert

Yasar Erdem

Richard Erdman

Georg Erhardt

Peter Erickson

Ronald Erskine

Jeffery Escobar

Angélica Espinoza-Ortega

David Everett

Jaap Evers

Willard Eyestone

Ephraim Ezra

Michele Faccia

Colette Fagan

Alan Fahey

Marie-Hélène Famelart

Nana Farkye

Frédéric Farnir

Harold Farrell

Bernard Faye

Vivek Fellner

Gene Felton

James Ferguson

Victoria Ferragut

José Bento Ferraz

Isabel Ferreira

Alfred Ferret

John Fetrow

Veerle Fievez

John Fike

Freddy Fikse

Ismail Filya

Raffaella Finocchiaro

Jeffrey Firkins

Richard Fitzgerald

Ross Fitzgerald

Steve Flint

Gonzalo Flores

Edward Foegeding
Leorges Fonseca

Luca Fontanesi

Monica Foote

Flavio Forabosco

Neil Forsberg

Lawrence Fox

Christian Frank

Joe Frank

Jose Fregonesi

Catja Freiburghaus

José Fresno

Joachim Frey

Gertraude Freyer

Paul Fricke

Theodore Friend

Nicolas Friggens

George Fthenakis

Carmen Fuentes

Birgit Fuerst-Waltl

Mohamed Gaber

Laurence Galet

K. Galvao

Bozhong Gan

J. Ignacio Garabal

Alvaro Garcia

Ian Gardner

Duane Garner

Phil Garnsworthy

Frank Garry

John Gaughan

Mathieu Gautier

John Gay

Gilda Gbassi

Tom Geary

Nicolas Gengler

Lia Gershenson

Rachel Gervais

Isabel Gigli

Robert Gilbert

Harsharn Gill

Warren Gilson

Christiane Girard

Elizabeth Glass

Sandra Godden

Lisbeth Goddik

Ann Godkin

John Goeser

H. Douglas Goff

Jesse Goff

Bruce Golden

Barry Goldin

Michael Gonda

Luciano González

Blanca Edelia González Martínez 
Oscar Gonzalez-Recio

Carlos Gonzalo

Robert Goodling

Elisha Gootwine

Patrick Gorden

Ronald Gorewit

Cameron Gourley

Nils Grabowski

Neville Grace

Kiev Gracias

Chris Grainger

A. S. Grandison

Richard Grant

Rick Grant

Sally Gras

Jane Martha Graton Mikcha

William Graves

Birgit Gredler

Alice Green

Martin Green

Paul Greenough

Pablo Gregorini

Tanya Gressley

Kenneth Griswold

Eildert Groeneveld

Yrjo Grohn

Daniel Grooms

Josef Gross

Ric Grummer

Xinfu Guan

Charles Guard

Daniel Guertin

Tim Guinee

Bernt Guldbrandtsen

Ahmet Gumen

Sanjay Gummala

Michael Gunderson

Mingruo Guo

Beatriz Gutierrez-Gil

Lorenz Gygax

Bianca Haase

David Habier

Tim Hackmann

Darryl Hadsell

George Haenlein

Sasha Hafner

Mekonnen Haile-Mariam

Ilan Halachmi

Mary Beth Hall

Elin Hallén

Stacey Hamilton

Hedi Hammami

Harald Hammon

Norziah Hani
Mark Hanigan

Alison Hanlon

Laura Hänninen

Leslie Hansen

Peter Hansen

David Harmon

Jan Harms

Lowry Harper

W. Harper

Keith Harris

Joe Harrison

Steven Hart

Federico Harte

Gary Hartnell

Kevin Harvatine

Marie Haskell

Ashraf Hassan

Ali Hayaloglu

Ben Hayes

Catherine Hayhurst

Armagan Hayirli

Fang He

Arlyn Jud Heinrichs

Steve Hendrick

T. Herdt

Bjorg Heringstad

Laura Hernandez

Victor Hernandez

Marta Hernandez-Jover

Humberto Hernandez-Sanchez

Mette Herskin

Gonzalo Hervas

Kasper Hettinga

Cord Heuer

Wolfgang Heuwieser

Peter Hildebrand

Arthur Hill

T. Hill

Eric Hillerton

Bradley Hilty

Dirk Hinrichs

Arnold Hippen

Gaby Hirsbrunner

Heidrun Hochwallner

Christina Hoelzel

Pat Hoffman

Joseph Hogan

Hendrik Hogeveen

William Hohenboken

Carl Holt

Kjell Holtenius

Menno Holzhauer

Brinton Hopkins

Brendan Horan
David Horne

Russell Hovey

Mari Hovinen

Ernest Hovingh

Wayne Howard

Alexander Hristov

Daniela Huessy

Pekka Huhtanen

Gerald Huntington

Thom Huppertz

Walter Hurley

Michael Hutjens

Juliana Huzzey

Erica Ruth Hynes

Salam Ibrahim

Giles Innocent

Ignacio Ipharraguerre

Richard Ipsen

Hidetomo Iwano

Barnouin Jacques

Shalini Jain

Robert James

Janusz Jamrozik

Lee Ann Jaykus

Thomas Jenkins

Bruce Jenny

Margit Bak Jensen

Zhihua Jiang

Rafael Jimenez-Flores

Kjell Johansson

Dave Johnson

Kristen Johnson

Mark Johnson

David Jones

Jamie Jonker

Ellen Jordan

Sergio Juchem

Jarmo Juga

Vincent Juillard

S. Julia

Hans-Joachim Jung

Darren Juniper

Haja Kadarmideen

Kasipathy Kailasapathy

Gonul Kaletunc

Kenneth Kalscheur

Hachiro Kamada

Rui Kano

Rohit Kapoor

Binnur Kaptan

Yonca Karagul Yuceer

Fatma Karakas Oguz

Elizabeth Karcher

Sanjay Karnati 
Jeffrey Karns

Niel Karrow

Ramanathan Kasimanickam

Martin Kaske

Reza Kasravi

Kazuo Kato

Kerry Kaylegian

Ermias Kebreab

Gregory Keefe

Elizabeth Kegley

Marcus Kehrli

Wayne Kellogg

Alan Kelly

Philip Kelly

David Kelton

Mary Keniry

David Kenny

Ronald Kensinger

Sandra Kentish

Jeffrey Keown

David Kerr

Alois Kertz

Amayun Khan

Ramesh Khanal

Suresh Kharche

Hasan Khatib

Mehar Khatkar

Christa Khn

Manfred Kietzmann

So Hyun Kim

Jennifer Kimmel

Hiromi Kimoto-Nira

Ronald Kincaid

Paul Kindstedt

Brian Kirkpatrick

Gerrit Kistemaker

Haruki Kitazawa

Soressa Kitessa

Joachim Kleen

Daryl Kleinschmit

Terry Klopfenstein

Joanne Knapp

Martin Knicky

Katharine Knowlton

Richard Kohn

Sven König

Paul Kononoff

Gerd Konrad

Gerrit Koop

Mikko Koskinen

Mahmoud Kouchak

Athanasios Koutinas

Karen Krause

P. Krawczel
Michael Kreuzer

Niels Kristensen

Michaela Kristula

Volker Krömker

Pasad Kulatunga

Hanna Kulig

Limin Kung

Clemens Kunz

Victor Kuri

Daniel Kusche

Hae-Soo Kwak

Ilias Kyriazakis

Nicola Lacetera

Christophe Lacroix

Olimpia Lai

Louis Laleye

Theo Lam

Graham Lamb

William Lamberson

W. Lamm

Amy Lammert

Christoph Lammler

Duane Larick

Mette Larsen

Mogens Larsen

Robert Larson

Alejandra Latorre

Richard Laven

Thomas Lawlor

Lartey Lawson

Yannick Le Cozler

Ian Lean

Stephen Leblanc

Sanghong Lee

Soo Lee

Alan Lefcourt

Andrés Legarra

Florian Leiber

Gabriel Leitner

Caleb Lemley

Sophie Lemosquet

Jo Leroy

Ken Leslie

Eva Lewis

Ronald M. Lewis

Anne Lichtenwalner

Manuele Liciardi

M. Lidauer

Lena Lidfors

Chen Lijun

Ole Lind

Raphael Linker

Min-Tze Liong

Kerry Lissemore
Willie Loeffen

Scott Loeffler

Jason Lombard

Juan Loor

Secundino Lopez

Mario Lopez-Benavides

Ivan Lopez-Exposito

Rosina Lopez-Fandiño

Marta Lourenco

John Lucey

Matthew Lucy

Mogens Lund

Anne Lundén

Jan Lundqvist

Tim Lysyk

Ying Ma

Sameer Mabjeesh

Nicolo Macciotta

Ruth Macdonald

Noirin Machugh

Keith Macmillan

Per Madsen

Elizabeth Maga

Rubén Maggio

David C. J. Main

Azwihangwisi Maiwashe

Aduli Enoch Malau-Aduli

Francisco Malcata

Jakob Malmo

Christian Maltecca

Ephraim Maltz

Michael Mangino

Soulaf Mansour

Esa Antero Mantysaari

C. Marce

Jeremy Marchant-Forde

Jean Margerison

Juan Marini

Marilena Marino

Massimo Mariscoli

Pierre-Guy Marnet

Milan Marounek

Cecile Martin

Greg Martin

Maria Martin

Patrice Martin

Sandra Martin Del Campo

Silvana Martini

Raluca Mateescu

L. Mateus

Ian Mather

Motozumi Matsui

Gilles Mauris

Manfred Mayer 
Baltasar Mayo

Sinéad Mc Parland

Alan McAllister

Jack McAllister

Craig McConnel

M. McCormick

Scott McDougall

Lee McDowell

Joseph McFadden

Lyndy McGaw

Robert McGorrin

Mark McGuire

Shelley McGuire

Sheila McGuirk

Paul McJarrow

John McKillip

Donald McMahon

William McManus

John McNamara

Margarita Medina

John Mee

Anne Megaro

Jalil Mehrzad

Bhavbhuti Mehta

Susanne Meier

Graeme Mein

Enoch Meira Junior

Marcello Mele

Dominic Mellor

Erdogan Memili

Uzi Merin

Cornelia Metges

Jos Metz

Lloyd Metzger

Theo Meuwissen

Heinrich Meyer

Mireille Meylan

John Middleton

Michal Milerski

Daniel Miller

David Miller

Michael Miller

Robert Miller

Mathieu Millette

Giulietta Minozzi

Tom Misselbrook

Ignacy Misztal

Frank Mitloehner

Makoto Mitsumori

Kerstin Mller

Adrian Molenaar

Pilar Molina

Eduarda Molina Alcaide

Joachim Molkentin
Juerg Moll

Hugo Montaldo

Sergio Monteagudo

Tera Montgomery

Jon Moorby

Chel Moore

Dale Moore

Ofelia Mora

Cesar Morales

James Moran

Stefano Morandi

Carmen Moraru

Diego Morgavi

Pierre Morin

Paolo Moroni

Amir Mortazavian

Gerhard Moser

Diego Moya

Kasey Moyes

Raphael Mrode

P. Mueller-Buschbaum

Christoph Muelling

Biswarup Mukhopadhyay

Herman Mulder

Isis Mullarky

Finbar Mulligan

Lene Munksgaard

Marcos Munoz

Michael Murphy

T. Nagaraja

Masoud Najafi

T. Nakao

Fabio Napolitano

Arjan Narbad

Wytze Nauta

Raymond Nebel

João Negrão

Riccardo Negrini

F. Neijenhuis

Brandon Nelson

Tamilee Nennich

Timothee Neuenschwander

Stefan Neuner

C. Jamie Newbold

Stephen Nickerson

Hanne Nielsen

S. Nielsen

Soeren Nielsen

Theo Niewold

Akbar Nikkhah

Yoshiaki Nishiya

Rauni Niskanen

Brian Nonnecke

Peder Noorgard
Kenneth Nordlund

H. Norman

Hubert Nowakowski

Anna Nudda

Manuel Nunez

Karl Nuss

George Nychas

Daryl Nydam

Ann-Kristin Nyman

Masahito Oba

John O'Callaghan

Michael O'Connor

Jørgen Ødegård

Liliana Odierno

Jack Odle

Nicholas Odongo

Michael O'Donovan

Keelin O'Driscoll

Garrett Oetzel

Shin Oikawa

Georgios Oikonomou

Richard O'Kennedy

Ammar Olabi

Richard Olde Riekerink

Carlos Oliveira

Katrina Olson

Timothy Olson

Pascal Oltenacu

James Oltjen

Nicole Olynk

Edgar Ontsouka

Charles Onwulata

Bonney Oommen

Geert Opsomer

Michael Orford

Henrik Østdal

Olav Osteras

Soren Ostergaard

Gernot Osthoff

Daniel O'Sullivan

Stephen Ott

Michael Overton

Thomas Overton

Max Paape

Colin Palmer

Donald Palmquist

Nikolaos Panousis

Paul Paquin

Young Park

E. Pärna

Peter Parodi

Matti Pastell

Sally Paulson

Ferenc Peles 
Luisa Pellegrino

Mauro Penasa

Yan Peng

Gregory Penner

Jodie Pennington

Kalyiani Perera

M. Ángeles Pérez-Cabal

Cristofol Peris

Vincent Perreten

George Perry

Karin Persson Waller

Augustine Peter

Ernst Peterhans

Robert Peters

Daniel Peterson

Lennart Petersson

Christina Petersson-Wolfe

Hélène Petit

Arun Phatak

Kaufman Phillip

Gina Pighetti

Anne Pihlanto

Roxanne Pillars

Paola Pilo

Jose Pires

Giacomo Pirlo

Jan Plaizier

Mario Podesta

Ioannis Politis

Geoffrey Pollott

Palmiro Poltronieri

Kayla Polzin

Yves Pouliot

Mark Powell

Frederick Provenza

Jennie Pryce

Pratap Pullammanappallil

Dan Putnam

Satu Pyorala

Xuejun Qiu

Muhammad Qureshi

Herman Raadsma

Roy Radcliff

D. Owen Rae

Eran Raizman

Päivi Rajala-Schultz

Maria Jose Ranilla

Scott Rankin

Jan Rasmussen

Morten Rasmussen

Oldemiro Rego

Shakeel Ur Rehman

Gary Reineccius

Douglas Reinemann
Tim Reinhardt

Martin Reist

Robert Rekawiecki

Boulbaba Rekik

Olav Reksen

Fazheng Ren

Jeffrey Reneau

Stefan Rensing

Christopher Reynolds

Pedram Rezamand

Michelle Rhoads

Fiona Rhodes

Carlos Risco

Ali Risvanli

Agustin Rius

Gilles Robitaille

John Roche

Luis Rodriguez

Maria Rodriguez-Prado

Uwe Roesler

C. Rontved

Anki Roth (Roxstrom)

C. Rotz

Maristela Rovai

Héctor Rubinstein

Pamela Ruegg

Claudia Ruiz-Capillas

Duncan Runciman

Rachel Rupp

Jeff Rushen

Jeffrey Rushen

Chris Russell

Zaeyoung Ryoo

Hassan Sadri

A. Sahoo

Roberto Sainz

Janeen Salak-Johnson

Ahmed Salama

Siti Isrina Salasia

Antonia Samoré

Bill Sanchez

Juan Sanchez

Margarita Sánchez Campos

Ashley Sanders

José-Eduardo Santos

Marcos Santos

Mehdi Sargolzaei

James Sartin

Roberto Sartori

Osamu Sasaki

Kan Sato

Kenji Sato

Charles Sattler

Helga Sauerwein
Ashish Sawant

Larry Schaeffer

Walter Schaeren

Flavio Schenkel

Anke Schennink

Siegfried Scherer

Irene Schiller

Wes Schilling

David Schingoethe

Paul Schlosser

Philippe Schmitt

Maria Del Pilar Schneider

Tonya Schoenfuss

Daniel Scholl

Jan Schonewille

Jon Schoonmaker

F. Schrick

Bernd Schroeder

J. Schroeder

Gustavo Schuenemann

Gertraud Schuepbach-Regula

Ynte Schukken

Nina Schulman

Michael Schutz

Charles Schwab

Harvey Scott

Karl-Heinz Sdekum

Philip Sears

Henri Seegers

George Seidel Jr.

Kris Sejrsen

Seongwon Seo

Sarah Serajzadeh

Eyal Seroussi

Asheber Sewalem

Hans-Martin Seyfert

Anthony Seykora

Roger Shanks

Bhawani Sharma

Vivek Sharma Sharma

Randy Shaver

Jan Shearer

Diarmuid Sheehan

Paul Sheehy

Martin Sheldon

Shannon Shields

Kei-Ichi Shimazaki

N. Shpigel

Henner Simianer

Peter Simko

Harjinder Singh

Kuljeet Singh

Siv Skeie

Betsie Slaghuis 
David E. Smith

Gilbert Smith

Julia Smith

K. Smith

Matt Smith

Michael Smith

Rebecca Smith

Stephen Smith

Warren Snelling

Gary Snowder

Sandro Soares

Åse Sogstad

Dagmar Solichova

Robert Soliva-Fortuny

George Somkuti

Man Song

Kamlesh Soni

A. Christian Sorensen

Jan Sorensen

Martin Sorensen

Michelle Soupir

Hélène Soyeurt

James Spain

Jerry Spears

Richard Spelman

Donald Spiers

Diane Spurlock

Judith Stabel

Katarzyna Stachowicz

Katharina Staerk

Charles Stallings

Eva Standarová

Edward Stanisiewski

Charles Staples

Michael Stear

Kerst Stelwagen

Roger Stephan

Marshall Stern

Jeffrey Stevenson

Marvin Stevenson

George Stilwell

Martin Stokes

Tracy Stokol

William Stone

Joseph Stookey

W. Marianne Stoop

Normand St-Pierre

Tomasz Strabel

Erling Strandberg

Ismo Stranden

Peter Strapak

Guosheng Su

Anand Subramanian

Peter Sullivan
Hector Sumano

Kerstin Svennersten-Sjaunja

Hermann H. Swalve

Janice Swanson

William Swecker

Matt Sylte

Joanna Szyda

Miika Tapio

Suvi Taponen

Loren Tauer

Luis Tedeschi

Assadollah Teimouri Yansari

Evgenij Telezhenko

Hasan Temiz

Bernd-Alois Tenhagen

Marta Terre

Georg Thaller

William Thatcher

Anne Thierry

David Thomas

Milton Thomas

Marvin Thompson

Paul Thompson

Peter Thomsen

Jennifer Thomson

Peter Thomson

Cameron Thraen

Leo Timms

Evan Titgemeyer

Nils Toft

Tadele Tolosa

Dana Tomlinson

Phillip Tong

Peter Tozer

Marko Tramsek

Philipp Trénel

Antonio-José Trujillo

Shogo Tsuruta

Cassandra Tucker

Michael Tunick

Anna-Maria Tyrisevä

Susanne Ulbrich

Dan Undersander

Zey Ustunol

Mette Vaarst

G. C. Vaillant

Georgios Valergakis

Roger Vallejo

Michael Van Amburgh

Sarel Van Amstel

Johan Van Arendonk

Leanne Van De Goor

Bart Van Den Borne

Rik Van Der Tol
Julius Van Der Werf

H. Van Dorland

Frank Van Eerdenburg

Diane Van Hekken

Koenraad Van Hoorde

Jan-Thijs Van Kaam

Jo Ann Van Kessel

H. Van Kruiningen

Isabelle Van Leuven

Gijs Van Ranst

Michael Vandehaar

Frédéric Vangroenweghe

Paul Vanraden

Gabriella Varga

Luis Varona

Ana Vazquez

Mercedes Vazquez-Anon

Roel Veerkamp

Douglas Veira

Isabelle Veissier

Drew Vermeire

Chris Verschoor

António Vicente

John Vicini

Johanna Vilkki

Beatriz Villanueva

E. Vlkova

Marina Von Keyserlingk

Atte Von Wright

Natascha Vukasinovic

Harit Vyas

Steinar Waage

Sarah Wagner

Dan Waldner

Matthew Waldron

Bill Wales

Anna Walker

Eileen Wall

Emma Wall

John Wallace

Hua Wang

Lei Wang

Yuxi Wang

Zhiquan Wang

Jeffrey Ward

Robert Ward

Steven Washburn

D. C. Wathes

Wes Watson

Rick Watters

Michel Wattiaux

William Wavrin

Daniel Weary

Miriam Weber Nielsen 
John Webster

Beat Wechsler

Neil Wedlock

Alfons Weersink

Mingken Wei

Kent Weigel

Rosmarie Weikard

Bart Weimer

Paul Weimer

Zwi Weinberg

William Weiss

Joel Weller

Olga Wellnitz

John Wenz

J. West

I. M. S. White

Robert Whitlock

Markus Wiederstein

George Wiggans

Diana Williams

Roderick Williams
David Wilson

Matthew Wilson

Milo Wiltbank

Christoph Winckler

Jack Windig

Wilhelm Windisch

Christopher Wolf

Eckhard Wolf

Wayne Wolf

David Wolfenson

Marie Wolfová

David Wood

Ewa Wredle

Tom Wright

M. Wszolek

Chao Wu

$\mathrm{Z}$. $\mathrm{Wu}$

Nicolas Wullepit

$\mathrm{Yu}$ Xia

Bob Xiang

Hariom Yadev
Wen Yang

Jesus Yaniz

Atilla YetIsmeyen

Allen Young

Fiona Young

Sara Youngerman

Ruth Zadoks

Tahir Zahoor Mian

Alfonso Zecconi

Shoushan Zeng

Steve Zeng

He-Ping Zhang

Lanwei Zhang

Feng-Qi Zhao

Xin Zhao

$\mathrm{Xu}$ Zhou

Malgorzata Ziarno

Clay Zimmerman

Richard Zinn

Bogdan Zisu

Birgit Zumbach 